\title{
THE USE OF ATTRACTIVE FIXED POINTS IN ACCELERATING THE CONVERGENCE OF LIMIT-PERIODIC CONTINUED FRACTIONS 1
}

\author{
JOHN GILL
}

ABSTRACT. A continued fraction can be interpreted as a composition of Möbius transformations. Frequently these transformations have powerful attractive fixed points which, under certain circumstances, can be used as converging factors for the continued fraction. The limit of a sequence of such fixed points can be employed as a constant converging factor.

The continued fraction

$$
\frac{a_{1}}{b_{1}}+\frac{a_{2}}{b_{2}}+\cdots+\frac{a_{n}}{b_{n}}+\cdots
$$

is said to be periodic in the limit provided $\lim a_{n}=a$ and $\lim b_{n}=b \neq 0$.

Set

$$
\begin{aligned}
& t_{n}(z)=a_{n} /\left(b_{n}+z\right), \quad n \geq 1, \\
& T_{1}(z)=t_{1}(z), \quad T_{n}(z)=T_{n-1}\left(t_{n}(z)\right), \quad n \geq 2,
\end{aligned}
$$

and

$$
\lim t_{n}(z)=t(z)=a /(b+z) .
$$

The $n$th approximant of (1) is obtained by setting $z=0$ in

$$
T_{n}(z)=\frac{a_{1}}{b_{1}}+\cdots+\frac{a_{n}}{b_{n}+z} .
$$

(1) is called periodic if $t_{n}(z) \equiv t(z), n \geq 1$; and if $t$ has two distinct fixed points, $u$ and $v$, where $|u| /|v|<1$, then one can write [1]

Received by the editors August 9, 1973.

AMS (MOS) subject classifications (1970). Primary 40A15; Secondary 40A25.

Key words and phrases. Limit-periodic continued fractions, converging factors, circles of Appollonius.

${ }^{1}$ This research was supported in part by AFOSR grant 70-1922. 


$$
\left(T_{n}(z)-u\right) /\left(T_{n}(z)-v\right)=(u / v)^{n}(z-u) /(z-v), \quad n \geq 1 .
$$

Clearly, $\lim T_{n}(z)=u$ for $z \neq v$.

An exact truncation error for a fixed $z$ follows easily from (3). For $z=$ 0 and $z=u$ this takes the forms

$$
T_{n}(0)-u=-u K^{n}(1-K) /\left(1-K^{n+1}\right), \quad n \geq 1,
$$

and

$$
T_{n}(u)-0 \equiv 0, \quad n \geq 1,
$$

where $K=u / v$.

Instant maximum acceleration of the periodic fraction occurs, therefore, upon replacing $z=0$ by $z=u$ in (2). Let $\left\{u_{n}\right\}$ and $\left\{v_{n}\right\}$ be the fixed points of $\left\{t_{n}\right\}$, chosen so that $\left|u_{n}\right| /\left|v_{n}\right|<1$. This paper is devoted to describing certain continued fractions, periodic in the limit, whose convergence may be speeded by setting $z=u$ or $z=u_{n+1}$ in $T_{n}(z)$. A geometrical approach leads to a priori truncation error estimates of $T_{n}(u)$ and $T_{n}\left(u_{n+1}\right)$. The technique is similar to that used in [2].

Previous articles associating the convergence behavior of continued fractions with the behavior of the sequences $\left\{u_{n}\right\}$ and $\left\{v_{n}\right\}$ include [2], [3] and [6]. Papers concerned with converging factors and/or contraction maps include [4] and [7].

Computations involving $u$ (or $u_{n+1}$ ) are accomplished as follows: Let $P_{n}$ and $Q_{n}$ be the $n$th partial numerator and $n$th partial denominator of (1) [5] so that $T_{n}(0)=P_{n} / Q_{n}, n \geq 1$. Let $P_{n}^{*}=P_{n}+u P_{n-1}, Q_{n}^{*}=Q_{n}+u Q_{n-1}$, $n \geq 2$. Then $T_{n}(u)=P_{n}^{*} / Q_{n}^{*}, n \geq 2$.

The phenomena of instantaneous convergence is not restricted to periodic fractions. Write $t_{n}$ in terms of its fixed points.

$$
t_{n}(z)=-u_{n} v_{n} /\left[-\left(u_{n}+v_{n}\right)+z\right], \quad n \geq 1 .
$$

Let $u_{n} \equiv u, \lim v_{n}=v$. Then (1) becomes

$$
\frac{u v_{1}}{u+v_{1}}-\frac{u v_{2}}{u+v_{2}}-\cdots-\frac{u v_{n}}{u+v_{n}}-\cdots
$$

Theorem 1. Let $T_{n}$ be defined in accordance with (6). If $0<|u|<\left|v_{n}\right|$, $n \geq 1$ and $|u|<|v|$, then $\lim T_{n}(0)=\lim T_{n}(u)=T_{n}(u) \equiv u$.

Proof. Theorem $1[6]$ implies $\left\{T_{n}(z)\right\}$ converges to a common limit for all $z \neq v . T_{n}(u) \equiv u$, since $t_{n}(u)=u, n \geq 1$. 
Theorem 2. Let $T_{n}$ be defined as before. If $u=v$ and $\Sigma\left|v_{n}-v_{n+1}\right|<$ $\infty$, then $\lim T_{n}(0)=\lim T_{n}(u)=T_{n}(u) \equiv u$.

Proof. Theorem 1 [3] guarantees the convergence of $\left\{T_{n}(z)\right\}$ to a common limit for every $z$.

It is well known that (1) converges provided $\lim \left(\left|u_{n}\right| /\left|v_{n}\right|\right)=|u| /|v|<1$. $\lim T_{n}(0)$ is near $u$ if $u_{n} \approx u, v_{n} \approx v$. The pattern of convergence is more complicated when $u=v$ or when $u \neq v$, but $|u|=|v|$. The first of these two special cases occurs in Theorem 2 .

In the three theorems that follow it is assumed that $u_{n} \rightarrow u \neq 0, v_{n} \rightarrow$ $v \neq 0,\left|u_{n}\right|<\left|v_{n}\right|$ and $|u|<|v|$, even though the last two restrictions may not always be necessary. Although having a formidable appearance, the hypotheses are not too difficult to satisfy.

Set

$$
\epsilon_{n}=\left|u_{n}-u\right| \cdot\left|u_{n}\right| /\left[\left|v_{n}\right|-\left|u_{n}-u\right|\right], \quad n \geq 1,
$$

and $H_{n}=\left|u_{n+1}-u_{n}\right|+\epsilon_{n+1}$.

Theorem 3. If

(i) $\left|u_{n}-u\right|>\left|u_{n}-u_{n+1}\right|$,

(ii) $\left|v_{n}\right|>2\left|u_{n}-u\right|$,

(iii) $\left|v_{n}\right| \geq\left|u_{n}-u\right|+\left|u_{n}-u\right| \cdot\left|u_{n}\right| /\left[\left|u_{n-1}-u\right|-\left|u_{n}-u_{n-1}\right|\right]$,

(iv) $\left|v_{n}\right|>\left|u_{n+1}-u_{n}\right|+\epsilon_{n+1}$

are all satisfied, then $\lim T_{n}(u)=\lim T_{n}\left(u_{n+1}\right)=\lim T_{n}(0)=T$, where $\left|T-u_{1}\right| \leq \epsilon_{1}$. Furthermore,

$$
2 \epsilon_{n} \prod_{1}^{n-1}\left\{\left|u_{j} v_{j}\right|\left[\left|u_{j+1}-u_{j}-v_{j}\right|-\epsilon_{j+1}\right]-2\right\}
$$

is an upper bound on both the errors $\left|T_{n}(u)-T\right|$ and $\left|T_{n-1}\left(u_{n}\right)-T\right|$. The monotonic divergence to 0 of the product is guaranteed by

(v) $\left|v_{n}\right|^{2}-\left|v_{n}\right|\left(\left|u_{n}\right|+2 H_{n}\right)+H_{n}^{2}>0, \quad n \geq 1$.

Proof of Theorem 3. $\left|K_{n}\right|=\left|u_{n} / \nu_{n}\right|<1,|K|<1$ imply $\lim T_{n}(z)=$ $\lim T_{n}(0)=T$ for $z \neq v[6$, Theorem 1]. The boundary of the disk

$$
C\left(\epsilon_{n}, u_{n}\right)=\left\{z:\left|z-u_{n}\right| \leq c_{n}\left|z-u_{n}-v_{n}\right|\right\}=\left\{z:\left|t_{n}(z)-u_{n}\right| \leq \epsilon_{n}\right\},
$$

with $\epsilon_{n}$ defined by (8), and

$$
0<c_{n}=\epsilon_{n} /\left|u_{n}\right|<1
$$

is a circle of Appollonius with respect to the points $u_{n}$ and $u_{n}+v_{n}$ (see 
the figure). Its center, $g_{n}$, and radius, $R_{n}$, are given by

$$
g_{n}=u_{n}-c_{n}^{2} v_{n}\left(1-c_{n}^{2}\right)-1
$$

and

$$
R_{n}=c_{n}\left|v_{n}\right|\left(1-c_{n}^{2}\right)^{-1} \text {. }
$$

The following conditions hold.

(1) $t_{n}\left(C\left(\epsilon_{n}, u_{n}\right)\right)=N\left(\epsilon_{n}, u_{n}\right)=\left\{z:\left|z-u_{n}\right| \leq \epsilon_{n}\right\}, n \geq 1$.

(2) $u \in C\left(\epsilon_{n}, u_{n}\right), n \geq 1$.

(3) $N\left(\epsilon_{n}, u_{n}\right) \subset C\left(\epsilon_{n-1}, u_{n-1}\right), n \geq 2$.

(1) follows from (8), (ii) and (10).

(2) is equivalent to $\left|g_{n}-u\right| \leq R_{n}, n \geq 1$, which, by (11) and (12) can be written

$$
\left|u_{n}-u-c_{n}^{2} v_{n}\left(1-c_{n}^{2}\right)^{-1}\right| \leq c_{n}\left|v_{n}\right|\left(1-c_{n}^{2}\right)^{-1} .
$$

This inequality follows from

$$
\left|u_{n}-u\right|+c_{n}^{2}\left|v_{n}\right|\left(1-c_{n}^{2}\right)^{-1}=c_{n}\left|v_{n}\right|\left(1-c_{n}^{2}\right)^{-1},
$$

which is equivalent to (8), if we assume (ii).

(3) can be written $\left|g_{n-1}-u_{n}\right|+\epsilon_{n} \leq R_{n-1}, n \geq 2$, which will follow if

$$
\left|u_{n-1}-u_{n}\right|+c_{n-1}^{2}\left|v_{n-1}\right|\left(1-c_{n-1}^{2}\right)^{-1}+\epsilon_{n} \leq c_{n-1}\left|v_{n-1}\right|\left(1-c_{n-1}^{2}\right)^{-1} \text {. }
$$

(i) and (ii) suffice to imply the equivalence of this last inequality to (iii).

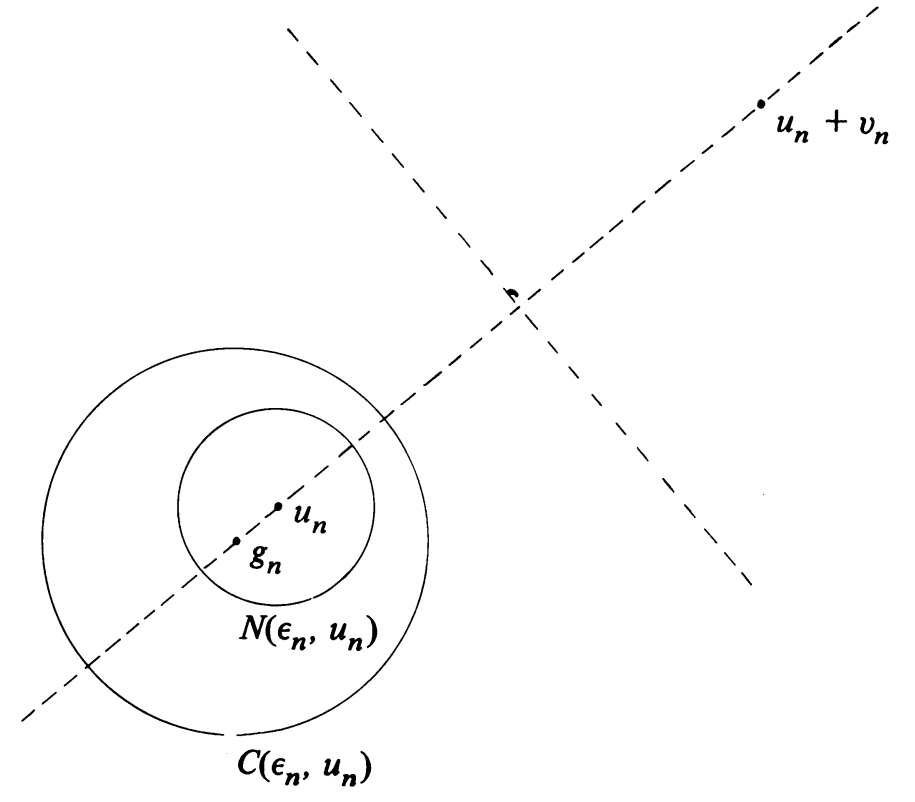


The contractive property of the $t_{n}$ 's now come into play. From (6) and the figure

$$
\begin{aligned}
\left|t_{n}(z)-t_{n}\left(z^{\prime}\right)\right| & =\frac{\left|u_{n} v_{n}\right| \cdot\left|z-z^{\prime}\right|}{\left|z-\left(u_{n}+v_{n}\right)\right| \cdot\left|z^{\prime}-\left(u_{n}+v_{n}\right)\right|} \\
& \leq \frac{\left|u_{n} v_{n}\right|}{\left(\left|u_{n+1}-u_{n}-v_{n}\right|-\epsilon_{n+1}\right)^{2}} \cdot\left|z-z^{\prime}\right|
\end{aligned}
$$

where $z$ and $z^{\prime} \in N\left(\epsilon_{n+1}, u_{n+1}\right)$. (iv) insures $\left|u_{n+1}-u_{n}-v_{n}\right|>\epsilon_{n+1}$. Thus the bound (9) is obtained. (v) makes the factors of the product less than one. The limit of these factors as $j \rightarrow \infty$ is $|u| /|v|<1$.

Let $\left\{\zeta_{n}\right\}$ be any sequence of points such that $\zeta_{n} \in N\left(\epsilon_{n}, u_{n}\right)$. Then $\lim T_{n-1}\left(\zeta_{n}\right)=\lim T_{n}(0)=T$, and $\left|T_{n-1}\left(\zeta_{n}\right)-T\right|$ is bounded by (9). An obvious choice of $\left\{\zeta_{n}\right\}$ is $\left\{u_{n}\right\}$.

This completes the proof of Theorem 3.

The hypotheses of Theorem 3 can be altered slightly to produce an alternate and possibly weaker form of (9).

Set

$$
\epsilon_{n}=\left|u_{n}-u\right|, \quad n \geq 1
$$

and

$$
l_{n}=\left|u_{n+1}-u_{n}\right|+\epsilon_{n+1}, \quad n \geq 1
$$

Theorem 4. If

(i) $0<\epsilon_{n}<\left|u_{n}\right|, \quad n \geq 1$,

(ii) $\left|v_{n}\right|>\left|u_{n}\right|+\epsilon_{n}, \quad n \geq 1$,

(iii) $\left|v_{n}\right| \geq\left(1+\left|u_{n}\right| \epsilon_{n}^{-1}\right) l_{n}, n \geq 1$,

are all satisfied, then $\lim T_{n}(u)=\lim T_{n}\left(u_{n+1}\right)=\lim T_{n}(0)=T$, where $\left|T-u_{1}\right| \leq \epsilon_{1}$. Furthermore,

$$
2 \epsilon_{n} \prod_{1}^{n-1}\left\{\left|u_{j} v_{j}\right|\left[\left|u_{j+1}-u_{j}-v_{j}\right|-\epsilon_{j+1}\right]-2\right\}
$$

is an upper bound on both the errors $\left|T_{n}(u)-T\right|$ and $\left|T_{n-1}\left(u_{n}\right)-T\right|$. The monotonic divergence to 0 of the product is guaranteed by

$$
\text { (iv) }\left|v_{n}\right|^{2}-\left|v_{n}\right|\left(\left|u_{n}\right|+2 l_{n}\right)+l_{n}^{2}>0, n \geq 1 \text {. }
$$

Remark. If $\epsilon_{n}(\downarrow)$ and $\left|u_{n+1}-u_{n}\right|<\epsilon_{n}$, then $\left|v_{n}\right|>2\left(\epsilon_{n}+\left|u_{n}\right|\right)$ implies (iii). 
Proof of Theorem 4. Conditions (1), (2), and (3) in the proof of Theorem 3 are satisfied assuming (i), (ii) and (iii) of Theorem 4. The details of proof are much the same.

Example 1. $\left[300\left(10^{-4 n}-1\right) /\left(20+10^{1-4 n}\right) i\right]_{n=1}^{\infty}$, where $u_{n}=10\left(1-10^{-4 n}\right) i$ $\rightarrow u=10 i$ and $v_{n} \equiv v=-30 i$. The hypotheses of Theorem 3 are satisfied.

$$
\begin{array}{ccccc}
\text { actual error: } & \left|T_{n}(0)-T\right| & \left|T_{n}(u)-T\right| & \left|T_{n}\left(u_{n+1}\right)-T\right| & \text { error bound } \\
N=2 & 1.4 & 1.4 \times 10^{-12} & 3.7 \times 10^{-15} & 2.2 \times 10^{-9} \\
N=3 & 5 \times 10^{-1} & 2.1 \times 10^{-15} & & \\
7.3 \times 10^{-14}
\end{array}
$$

Note that the error bound for $\left|T_{2}\left(u_{3}\right)-T\right|$ is given in the second row of figures.

The hypotheses of Theorem 3 can be changed, in the following special case without affecting the error bound (9). Replace $b_{n}$ by 1 in (1).

Set

$$
\epsilon_{n}=\left|u_{n}\right|\left(u_{n}-u\right) /(u+1), \quad n \geq 1
$$

Theorem 5. If

(i) $u_{n}$ and $u \neq-1,-1$ lie on a ray, and $u$ is between $u_{n}$ and -1 ,

(ii) $\left|u_{n}-u\right|(\downarrow)$,

(iii) $\left|u_{n}-u\right|<|u-1|$,

(iv) $\left|u_{n}\right|<|u+1|$

are all satisfied, then $\lim T_{n}(u)=\lim T_{n}\left(u_{n+1}\right)=\lim T_{n}(0)=T$, where

$$
\left|T-u_{1}\right| \leq\left|u_{1}\right| \cdot\left|u_{1}-u\right| /\left|2 u_{1}-u+1\right| \text {. }
$$

Furthermore,

$$
2 \epsilon_{n} \prod_{1}^{n-1}\left\{\left|u_{j}\left(u_{j}+1\right)\right|\left[\left|u_{j+1}+1\right|-\epsilon_{j+1}\right]^{-2}\right\}
$$

is an upper bound on both the errors $\left|T_{n}(u)-T\right|$ and $\left|T_{n-1}\left(u_{n}\right)-T\right|$.

Proof. Observe that $v_{n}=-u_{n}-1$ and $v=-u-1$.

(1) follows from (i) and (iii).

(2) is satisfied if $\left|g_{n}-u\right|=R_{n}$. A brief computation shows that this is equivalent to (15). (ii) implies $\left|g_{n-1}-u\right| \geq\left|g_{n}-u\right|$, which guarantees $C\left(\epsilon_{n}, u_{n}\right) \subset C\left(\epsilon_{n-1}, u_{n-1}\right)$. (iv) is equivalent to $N\left(\epsilon_{n}, u_{n}\right) \subset C\left(\epsilon_{n}, u_{n}\right)$. Hence (3) is satisfied.

Example 2. Set $F(x)=(x / \operatorname{Arctan} x)-1$, where [5, p. 116] 


$$
\operatorname{Arctan} x=\frac{x}{1}+\frac{x^{2}}{3}+\cdots+\frac{n^{2} x^{2}}{2 n+1}+\cdots \cdot
$$

An equivalence transformation on $F$ gives

$$
F(x)=\left[\frac{n^{2} x^{2} /\left(4 n^{2}-1\right)}{1}\right]_{n=1}^{\infty} .
$$

The hypotheses of Theorem 5 are satisfied for $x=\sqrt{3}$.

$$
\begin{array}{ccccc}
\text { actual error: } & \left|T_{n}(0)-T\right| & \left|T_{n}(u)-T\right| & \left|T_{n}\left(u_{n+1}\right)-T\right| & \text { error bound (16) } \\
n=2 & 9.8 \times 10^{-2} & 1.8 \times 10^{-3} & 2.1 \times 10^{-4} & 7.5 \times 10^{-3} \\
n=3 & 3.5 \times 10^{-2} & 3.3 \times 10^{-4} & 3.1 \times 10^{-5} & 1.1 \times 10^{-3} \\
n=4 & 1.1 \times 10^{-2} & 7.0 \times 10^{-5} & & \\
2.1 \times 10^{-4}
\end{array}
$$

Although the theorems in this paper can be applied to any type continued fraction, frequently the fixed points have complicated structures. The following formal procedure can be used to convert a power series into a "fixed point type" fraction.

Let $P(z)=1+a_{1}^{(1)} z+a_{2}^{(1)} z^{2}+\cdots$. Write

$$
\begin{gathered}
P(z)=1+c_{1} z^{k^{1}}\left(1+b_{n_{1}}^{(1)} z^{n_{1}}+\cdots\right), \quad k_{1} \geq 1, n_{1} \geq 1, \\
c_{1}=d_{k_{1}}^{(1)}, \quad k_{1}=\min \left\{n: a_{n}^{(1)} \neq 0\right\} .
\end{gathered}
$$

Then

$$
\begin{aligned}
P(z) & =1+\frac{c_{1} z^{k_{1}}}{1+a_{1}^{(2)} z+a_{2}^{(2)} z^{2}+\cdots} \\
& =1+\frac{c_{1} z^{k_{1}}}{1+c_{1} z^{k_{1}}-c_{2} z^{k^{k}}\left(1+b_{n_{2}}^{(2)} z^{n_{2}}+\cdots\right)}, \text { etc. }
\end{aligned}
$$

This gives rise to the fraction

$$
1-\mathrm{K}_{n=1}^{\infty}\left(\frac{-c_{n} z^{k_{n}}}{1+c_{n} z^{k_{n}}}\right),
$$

with fixed points $u_{n}=c_{n} z^{k_{n}}, v_{n} \equiv 1$. Observe that (17) appears in the equivalent continued fraction expansion of 


$$
Q(z)=1+(2-P(z))^{-1}=1+1+c_{1} z^{k_{1}}+\cdots .
$$

A purely formal application to $e^{x}, x=1 / 10$, gives

$$
\begin{array}{cc}
\text { actual error: } & \left|T_{n}\left(u_{n+1}\right)-e^{1 / 10}\right| \\
n=2 & 5 \times 10^{-7} \\
n=3 & 1 \times 10^{-8}
\end{array}
$$

\section{REFERENCES}

1. L. R. Ford, Automorphic functions, McGraw-Hill, New York, 1929.

2. J. Gill, Attractive fixed points and continued fractions, Math. Scand. 33 (1973), 261-268.

3. - Infinite compositions of Möbius transformations, Trans. Amer. Math. Soc. 176 (1973), 479-487.

4. T. Hayden, Continued fraction approximation to functions, Numer. Math. 7 (1965), 292-309. MR $32 \# 3258$.

5. A. N. Hovanskii, The application of continued fractions and their generalizations to problems in approximation theory, GITTL, Moscow, 1956; English transl., Noordhoff, Groningen, 1963. MR 27 \#6058.

6. M. Mandell and A. Magnus, On convergence of sequences of linear fractional transformations, Math. Z. 115(1970), 11-17. MR 41 \#3621.

7. P. Wynn, Converging factors for continued fractions. I, II, Numer. Math. 1 (1959), 272-320. MR 22 \#6953.

DEPARTMENT OF MATHEMATICS, SOUTHERN COLORADO STATE COLLEGE, PUEBLO, COLORADO 81005 